\title{
Autonomous Analyser Platforms for Remote Monitoring of Water Quality
}

\author{
Dermot Diamond, John Cleary, Damien Maher, Jungho Kim, King Tong Lau \\ CLARITY Centre for Sensor Web Technologies, National Centre for Sensor Research, Dublin City University, Dublin 9, \\ Ireland. \\ E-mail: dermot.diamond@dcu.ie
}

\begin{abstract}
This paper describes progress in the realization of reliable, relatively low-cost autonomous microfluidic analysers that are capable of monitoring the chemistry of water bodies for significant periods of time (weeks, months) without human intervention. The data generated is transmitted wireless to a remote web server and transferred to a web-database that renders data access location independent. Preliminary results obtained from a 'matchbox' scale analyzer are also presented and routes to the realization of next generation platforms discussed.
\end{abstract}

Keywords: Microfluidics, water quality, environmental monitoring, chemical analyzers, nutrients.

\section{INTRODUCTION}

In an ideal world, the quality of our environment would be monitored in real time at multiple locations for a wide variety of key target analytes, and the information processed and made accessible in appropriate form for multiple stakeholders, including environmental specialists, industry staff, Government agencies, and society in general. The reality is unfortunately still far removed form this ideal, due in main to the cost of providing fit-for-purpose sensing technologies, and particularly, devices capable of providing information about the chemical and biological status of the environment. In this paper, we will focus on the design and functionality of platforms we have developed for monitoring water quality, and although the focus will be on reagent-based nutrient sensing, the approach is generic and can be applied to many important field measurements that can be achieved using reagent-based chemistries. It should also be noted that the platform technology we have developed has significant commonality for gas/air monitoring, particularly with respect to the electronics, enclosure, communications, and web-based data handling and visualization.

The overall goal of this research is to develop and field deploy analytical instruments that can monitor some aspect of the chemistry of a target water body for a substantial period of time (weeks, months). Furthermore, these devices should be cost effective to buy and operate, and function essentially in a 'deploy and forget' mode. Currently, we are able to provide a robust, functioning platform for under $€ 200$ (parts cost only) that is capable of performing 1,000-2,000 assays per $100 \mathrm{~mL}$ of reagent, due to the small volumes involved in microfluidic-based measurements.

Clearly, a key requirement for long-term deployments of autonomous analysers is reagent stability. Therefore, for the phosphate platform described below, we have therefore focused on the molybdovanadophosphoric 'yellow' method (reagents are very stable), rather than the more sensitive molybdate 'blue' method, which involves a reduction step to generate the intense blue complex (reagents less stable, and precipitations can form) [1].

\section{Autonomous ANAlyticAl PlatForms}

\section{A. Nutrient Analyser Platform}

Our work on the development autonomous nutrient analyser platforms for phosphate and ammonia began over ten years ago $[1,2]$. This laid the foundations for the development of the more recent autonomous field deployable nutrient analyser platforms. The first generation system [3] has been extensively field trialed both at wastewater treatment plants [4] and in natural waters [5]. Table 1 summaries the extensive field trials carried out using the first generation phosphate unit over the past 5 years, with over 9500 data points collects across 12 separate trials. Current work is focusing on the development of a second generation of the analyzer platform, which combines a 10 -fold lower cost with improved performance. Encouraging initial short-term field trials of this second generation system have been carried out in natural waters [6]. In this paper, we report a longer-term validation of the system, through a 23-day field trial at a wastewater treatment plant.

\section{B. First, second and next generation systems}

The first and second-generation phosphate analysers are shown in Figure 1. A detailed description of the first generation system design can be found in reference [7]. The second-generation system was designed as a more commercial version of the first generation system. Significant size, cost and weight reductions were achieved with the system redesign, while maintaining or improving system performance. Although the second-generation system has been designed around the phosphate chemistry, it can be adapted to accommodate the reagents and detection systems for other target analytes. Prototype systems have also been developed for the detection of nitrate/nitrite and ammonium and these are currently being integrated into field deployable platforms. These systems are also based on colorimetric detection, using the Griess reaction for nitrate/nitrite detection and the Berthelot (indophenol) reaction for ammonia detection. 


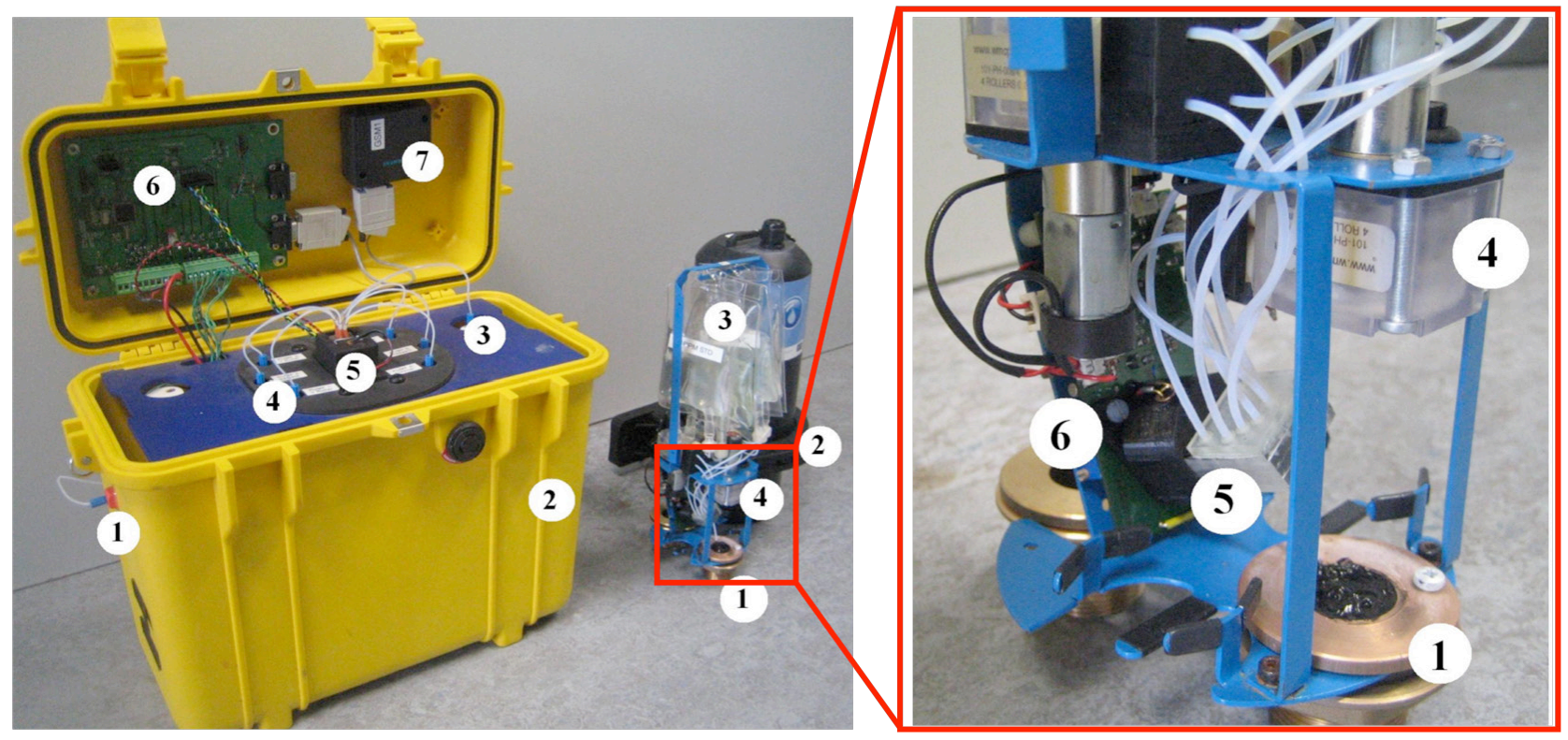

Figure 1: First and second generation phosphate systems. (1) Sample inlet; (2) IP68 Enclosure; (3) Reagent storage; (4) Pumps; (5) Microfluidic detection system; (6) Control board; (7) Communications.

TABLE I:

NUTRIENT ANALYSER FIELD TRIAL SUMMARY

\begin{tabular}{|c|c|c|c|c|}
\hline \multicolumn{5}{|c|}{ First Generation System } \\
\hline $\begin{array}{c}\text { Trial } \\
\text { Number }\end{array}$ & Date & Location & $\begin{array}{c}\text { Data points } \\
\text { collected }\end{array}$ & $\begin{array}{c}\text { Duration } \\
\text { (Days) }\end{array}$ \\
\hline 1 & $03 / 10 / 2006$ & Osberstown WWTP, Kildare, Ireland & 431 & 18 \\
\hline 2 & $01 / 05 / 2008$ & Inniscarra Reservoir, Cork, Ireland & 313 & 15 \\
\hline 3 & $30 / 06 / 2008$ & Osberstown WWTP, Kildare, Ireland & 1075 & 45 \\
\hline 4 & $01 / 10 / 2008$ & Osberstown WWTP, Kildare, Ireland & 1334 & 28 \\
\hline 5 & $10 / 11 / 2008$ & Osberstown WWTP, Kildare, Ireland & 3557 & 74 \\
\hline 6 & $27 / 11 / 2008$ & Osberstown WWTP, Kildare, Ireland & 992 & 21 \\
\hline 7 & $23 / 04 / 2009$ & Broadmeadow Water, Dublin, Ireland & 25 & 1 \\
\hline 8 & $26 / 05 / 2009$ & Broadmeadow Water, Dublin, Ireland & 100 & 4 \\
\hline 9 & $08 / 07 / 2009$ & Broadmeadow Water, Dublin, Ireland & 819 & 34 \\
\hline 10 & $04 / 09 / 2009$ & Rolestown Reservoir, Dublin, Ireland & 238 & 10 \\
\hline 11 & $25 / 09 / 2009$ & Broadmeadow Water, Dublin, Ireland & 195 & 8 \\
\hline 12 & $14 / 10 / 2009$ & Broadmeadow Water, Dublin, Ireland & 575 & 24 \\
\hline \multicolumn{5}{|c|}{ Second Generation System } \\
\hline 13 & $02 / 09 / 2010$ & Broadmeadow Water, Dublin, Ireland & 5 & 1 \\
\hline 14 & $04 / 09 / 2010$ & Broadmeadow Water, Dublin, Ireland & 124 & 3 \\
\hline 15 & $24 / 02 / 2011$ & Osberstown WWTP, Kildare, Ireland & 550 & 23 \\
\hline 16 & $07 / 04 / 2011$ & Osberstown WWTP, Kildare, Ireland & - & - \\
\hline & & Total & 10457 & 309 \\
\hline
\end{tabular}



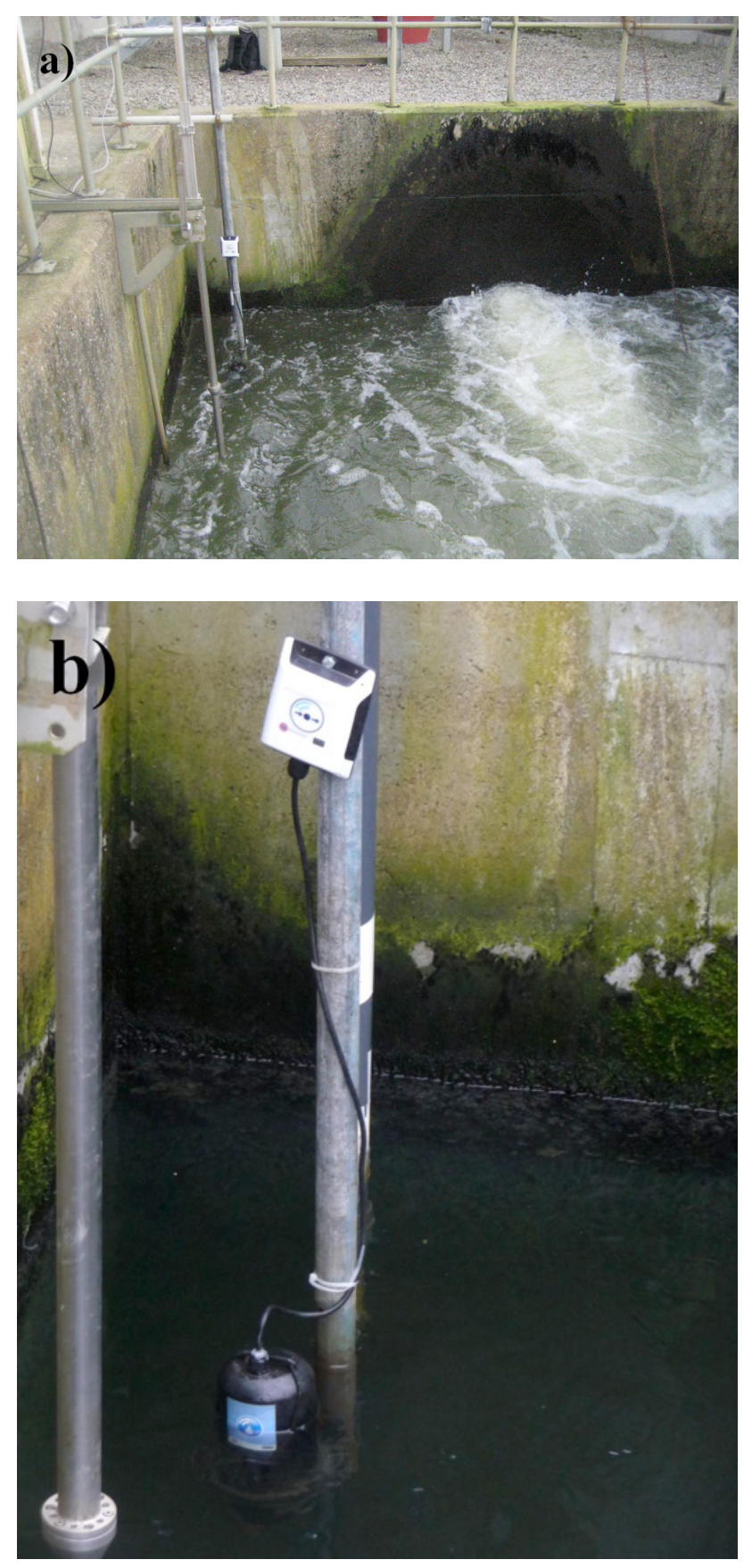

Figure 2: a) Effluent outflow tank, b) Phosphate analyser located below the water line and linked by cable to the communications module above.

\section{SECOND GENERATION FIELD TRIAL}

Osberstown wastewater treatment plant, Co. Kildare, Ireland was chosen as a site to conduct a long-term validation of the system. The analyser was located in the plant outflow tank (Figure 2) and setup to take hourly samples. The main analyser body was submerged, while the separate ZigBee radio communications module was located above the water surface, connected by cable to the analyzer. After each measurement, the ZigBee radio transmitted the data back to a network gateway and local database located on site. Remote login to the database allowed data visualisation and remote control of the analyser sampling rate.

The data reported by the analyser along with laboratory analysed samples is shown in Figure 3. Approximately 30 erroneous data points caused by air bubbles in the microfluidics have been removed from the data set. Parallel manual samples were analysed for subsequent validation in the laboratory using a Hach-Lange DR890 Portable Colorimeter and the appropriate reagent pack for phosphate analysis. The values obtained via this method are in good agreement with those reported by the phosphate analyser.

A daily pattern was also observed in the phosphate levels, which generally lie in the range 0.5 to $2.5 \mathrm{mg} / \mathrm{L}(\mathrm{ppm})$. Regular spikes in the phosphate concentration occur at approximately midnight during this daily cycle. Identification of events of this type can only be achieved with the temporal resolution provided by an autonomous monitoring system such as the phosphate analyzer operating at an appropriate sampling frequency. The plant management attributes the daily fluctuations in the phosphate level to sequenced inputs from feeder pumping stations. Clearly, these relatively low-cost autonomous (battery powered) analysers have the capability to provide rich information about the dynamics to water treatment processes, and of fluctuations in environmental water chemistry generally. The remote programmability of the platform also means that the sampling rate can be controlled remotely, to enable the best use of the fixed number of assays available from the reagent pack (typically 1,000-2,000). The fastest sampling rate (1020 minutes, depending on local temperature) is determined by the sample throughput, which includes washing and calibration cycles.

\section{AUTONOMOUS NitRITE ANALYSER}

Testing of the nitrite reagent chemistry and detection system has been carried out using the prototype set-up shown in Figure 4 (Top Left). The system consists of two micro pumps, microfluidic mixing and detection chips, electronic control board and wireless communications. The detection system consists of a $540 \mathrm{~nm}$ light emitting diode (LED) and photodiode. The micro-pumps deliver nitrite sample and Griess reagent through the mixing chip to the detection chip. The mixture is allowed to react for two minutes before the LED and photodiode are used to take an absorbance measurement across a $1.5 \mathrm{~mm}$ square channel in the detection chip. The absorbance of the reacted sample is proportional to the nitrite concentration in the original sample

The system was tested using five solutions with concentrations ranging from $0.0 \mathrm{mg} / \mathrm{L}$ to $0.6 \mathrm{mg} / \mathrm{L}$. The results of the analysis are shown in Figure 4 (bottom left). The error bars (hidden by the point symbols) represent \pm first standard deviation with $\mathrm{n}=5$ replicates for each 


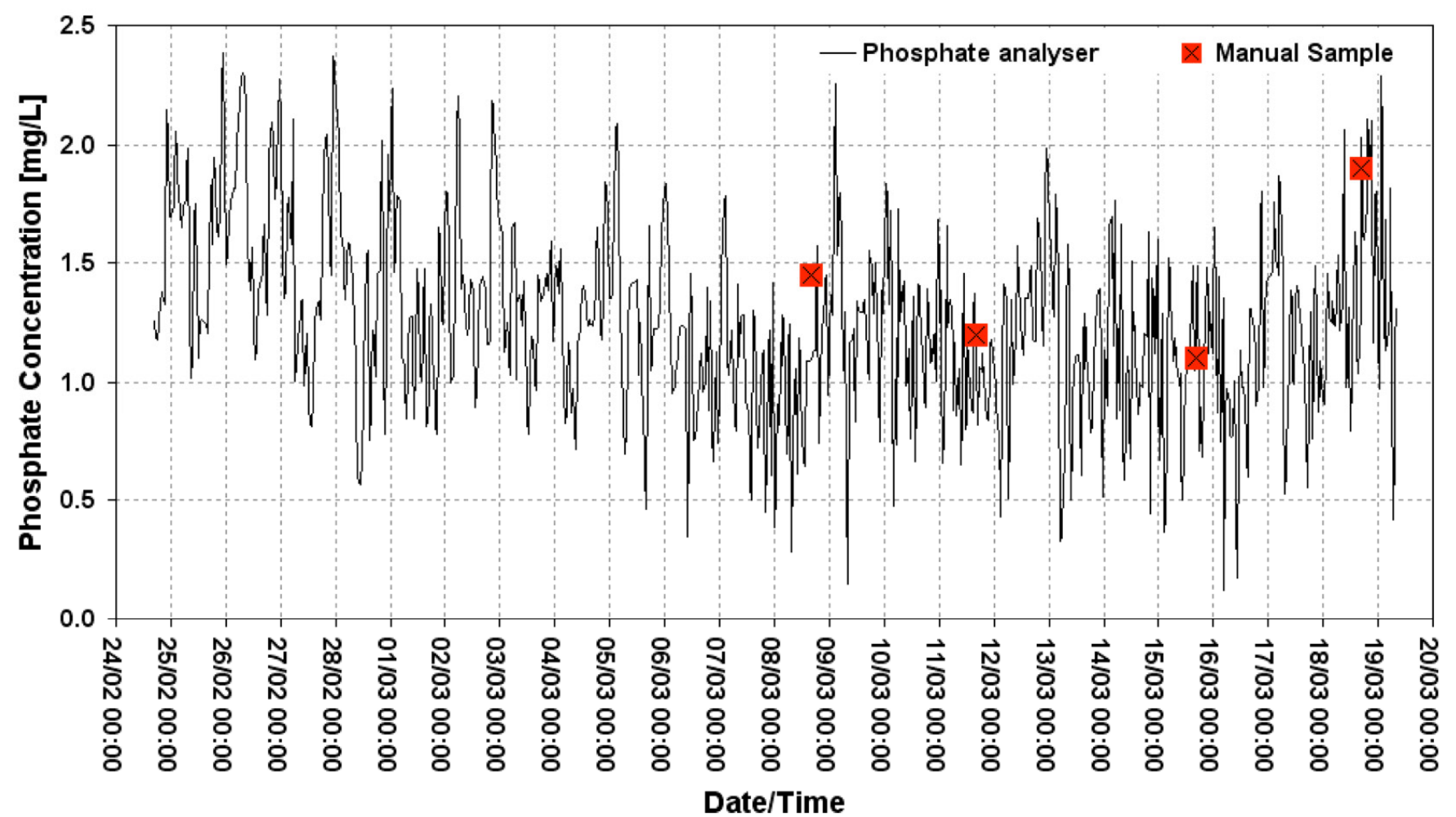

Figure 3: Data from the phosphate analyser and manual calibration samples (red) during the trial commencing 24/02/2011. Regular fluctuations arise from switching of local feeder pumping stations

determination. Based on this data, the detection limit of the system was estimated to be approximately $0.003 \mathrm{mg} / \mathrm{L}(3.0$ $\mathrm{ppb}$ ). This platform has excellent promise given the excellent sensitivity, limit of detection and reproducibility, although further work to determine the full linear range is still to be completed. Particularly important is the short timescale required to reach steady state signal for the samples (2 minutes) under laboratory conditions. Further development of the nitrite analyzer is continuing, with a particular emphasis on the inclusion of a reduction column to enable sample nitrate to be reduced in-line to nitrite. Switching the sample line alternatively from a direct channel to the Griess reagent and detector, to a channel bringing the sample through the reduction column to the reagent and detector enables sample nitrate to be estimated (nitrate $=$ total nitrite background nitrate).

\section{THE 'MATChBOX’ ANALYSER}

Work has also begun on the development of the next generation analyser platform, the so-called 'matchbox' analyser. A prototype system has been developed and tested [8]. The system, shown in Figure 4 (top right), uses a combination of polymer-actuated pumps and a paired emitterdetector light emitting diode detection system [9] to achieve a very low cost and compact platform. The polymer pumps are based on polypyrrole 'bender' actuators integrated into pump chambers that function as flexible diaphragms capable of driving liquid flow through the fluidic system [10]. In principle, these 'biomimetic' pumps are inherently more compatible with miniature analysers, and could be fully integrated into the fluidic platform, rather than as separate units as shown in figure 4 . Some preliminary analytical data (figure 4, bottom right) for nitrite via the Griess method shows that the system can produce real data, albeit of a reduced quality compared to the prototype analyzer (figure 4, bottom left). Nevertheless, it is encouraging that this 'matchbox' platform can generate analytical data of some use.

\section{CONCLUSIONS}

Low-cost autonomous analyzers capable of performing multiple assays in real field situations have been developed and validated. While the current platform is configured for phosphate analysis, suitable reagent-based methods for nitrite (and hence nitrate) and ammonia are available that can be employed in long-term developments (weeks, months). Extension to other environmentally important targets that can be analysed using colorimetric reagent methods should be relatively simple to implement.

A futuristic 'matchbox' version of the reagent analyzer has been demonstrated. However, significant challenges remain to be overcome before such platforms become reliable enough to meet the demands of the real-world. Perhaps the most important is the need for dramatic improvements in the 

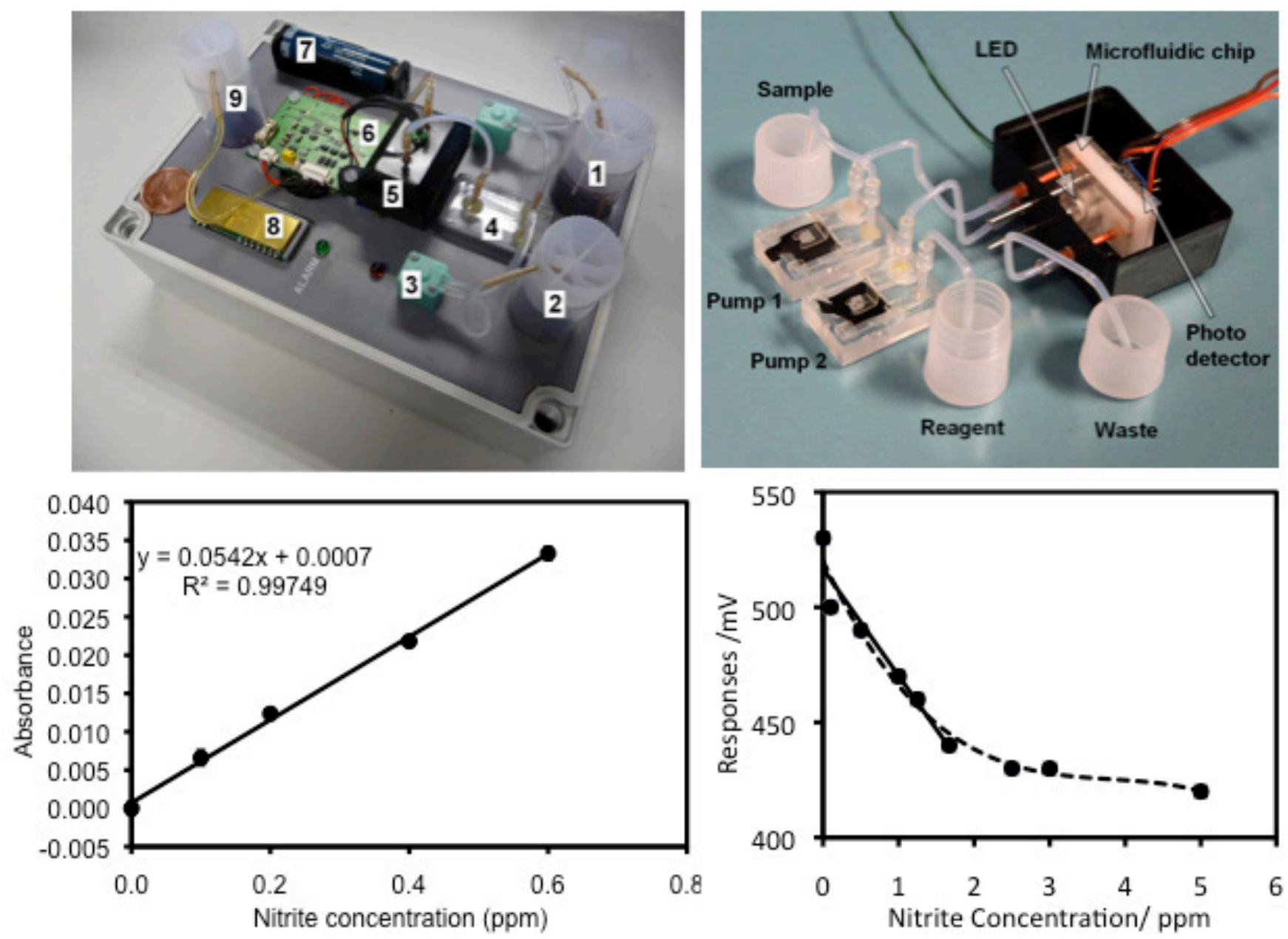

Figure 4: Top Left: Prototype nitrite analyzer based on the Griess Method. (1) Reagent storage (2) Sample storage (3) Micro-pump (4) Mixing chip (5) Detector (6) Control board (7) Battery (8) Easy-Radio (9) Waste storage.

Bottom Left: Typical data for nitrite standards obtained using the prototype nitrate analyzer showing excellent linerarity and sensitivity

Top Right: The futuristic 'Matchbox' Analyser showing component parts

Bottom Right: Preliminary data for nitrite standards obtained with the 'Matchbox' Analyser

reliability and lifetime of the polymer actuators. This is the subject of very considerable research effort [11], and perhaps breakthroughs in materials science, such as the emergence of effective photopolymer actuators for valves [12] and motors [13], will provide us with the basis tools and technologies to produce a 'tipping point' that will bring these potentially disruptive technologies into mainstream of environmental monitoring instrumentation.

\section{ACKNOWLEDGMENT}

We acknowledge support for this research from Science Foundation Ireland through the CLARITY Centre (grant code 07/CE/I1147), Enterprise Ireland (grant codes IP/2008/544 and CFTD/08/111). We also acknowledge support from Episensor Ltd. in project IP/2008/544.

\section{REFERENCES}

[1] M. Sequeira, M. Bowden, E. Minogue and D. Diamond, 'Towards Autonomous Environmental Monitoring Systems', Talanta, 56, Issue 2, pp. 355-363, 2002.

[2] M. Sequeira and D. Diamond, A. Daridon, J. Lichtenberg, S. Verpoorte, N. F. de Rooij, Progress in the realisation of an autonomous environmental monitoring device for ammonia, TrAC Trends in Analytical Chemistry, vol. 21, pp. $816-827,2002$.

[3] C. M. McGraw, S. E. Stitzel, J. Cleary, C. Slater, D. Diamond, 'Autonomous microfluidic system for phosphate detection', Talanta, Vol. 71, Issue 3, pp. 1180-1185, 2007.

[4] J. Cleary, C. Slater, C. McGraw, D. Diamond, 'An Autonomous Microfluidic Sensor for Phosphate: On-Site Analysis of Treated Wastewater', Sensors Journal, IEEE, vol.8, no.5, pp. 508-515, 2008.

[5] J. Cleary, D. Maher, C. Slater, D.Diamond, 'In situ monitoring of environmental water quality using an autonomous microfluidic sensor', Sensors Applications Symposium (SAS), Limerick, Ireland, pp. 36-40, 2010 .

[6] F. Collins, D. Orpen, D. Maher, J. Cleary, C. Fay and D. Diamond, 'Distributed Chemical Sensor Networks for Environmental Sensing', The Second International Conference on Sensor Device Technologies and Applications (SENSORDEVICES 2011), Côte d'Azur, France, August 21-27, 2011 (Submitted). 
[7] C. Slater; J. Cleary; C. M. McGraw; W. S. Yerazunis; K. T. Lau; D. Diamond, Autonomous field-deployable device for the measurement of phosphate in natural water, Advanced Environmental, Chemical, and Biological Sensing Technologies V, Proceedings of SPIE, Vol 6755, 2007, DOI: 10.1117/12.733754.

[8] J. H. Kim, K. T. Lau, R. Shepherd, Y. Wu, G. Wallace, D. Diamond, Performance characteristics of a polypyrrole modified polydimethylsiloxane (PDMS) membrane based microfluidic pump, Sensors and Actuators A: Physical, Vol. 148, Issue 1, pp. 239-244, 2008 .

[9] K.T. Lau, S. Baldwin, M. O'Toole, R. Shepherd, W. J. Yerazunis, S. Izuo, S. Ueyama, D. Diamond, A low-cost optical sensing device based on paired emitter-detector light emitting diodes, Analytica Chimica Acta, Vol. 557, Issues 1-2, pp. 111-116, 2006.

[10] J. Kim, K. T. Lau, C. Fay and D. Diamond, 'Development of optical sensing system for detection of $\mathrm{Fe}$ ions using conductive polymer actuator based microfluidic pump', IEEE Sensors, 26-29 October 2008, Lecce, Italy, paper B4P-L2, pp1155-1159; 2008, download available from http://doras.dcu.ie/711/.

[11] T. Mirfakhra, J.D.W. Madden, R.H. Baughman, 'Polymer artificial muscles', Materials Today, Vol. 10, Issue: 4, pp: 30-38, 2007

[12] F. Benito-Lopez, R. Byrne, A. M. Raduta, N. E. Vrana, G. McGuinness, D. Diamond, 'Ionogel-based light-actuated valves for controlling liquid flow in micro-fluidic manifolds', Lab Chip, 10 pp. 195-201, 2010.

[13] 'M. Yamada, M. Kondo, J.Mamiya, Y. Yu, M. Kinoshita, C. J. Barrett and T. Ikeda, Photomobile Polymer Materials: Towards Light-Driven Plastic Motors', Angew. Chem. Int. Ed., vol. 47, pp. 4986 -4988, 2008. 